\title{
Performance comparison of phenotypic and molecular methods for detection and differentiation of Candida albicans and Candida dubliniensis
}

Suhail Ahmad*, Ziauddin Khan, Mohammad Asadzadeh, Ajmal Theyyathel and Rachel Chandy

\begin{abstract}
Background: Candida albicans is the most pathogenic Candida species but shares many phenotypic features with Candida dubliniensis and may, therefore, be misidentified in clinical microbiology laboratories. Candidemia cases due to $C$. dubliniensis are increasingly being reported in recent years. Accurate identification is warranted since mortality rates are highest for C. albicans infections, however, C. dubliniensis has the propensity to develop resistance against azoles more easily. We developed a duplex PCR assay for rapid detection and differentiation of C. albicans from C. dubliniensis for resource-poor settings equipped with basic PCR technology and compared its performance with three phenotypic methods.
\end{abstract}

Methods: Duplex PCR was performed on 122 germ tube positive and 12 germ tube negative isolates of Candida species previously identified by assimilation profiles on Vitek 2 ID-YST system. Typical morphologic characteristics on simplified sunflower seed agar (SSA), and reaction with a commercial (Bichro-Dubli) latex agglutination test were also performed. The assay was further applied on 239 clinical yeast and yeast-like fungi and results were confirmed by DNA sequencing of internal transcribed spacer (ITS) region of rDNA.

Results: The results of duplex PCR assay for 122 germ tube positive and 12 germ tube negative isolates of Candida species were comparable to their identification by Vitek 2 ID-YST system, colony characteristics on SSA and latex agglutination test. Application of duplex PCR also correctly identified all 148 C. albicans and 50 C. dubliniensis strains among 239 yeast-like fungi.

Conclusions: The data show that both, duplex PCR and Bichro-Dubli are reliable tests for rapid (within few hours) identification of clinical yeast isolates as $C$. dubliniensis or $C$. albicans. However, duplex PCR may be applied directly on clinical yeast isolates for their identification as $C$. dubliniensis or $C$. albicans as it does not require prior testing for germ tube formation or latex Candida agglutination.

Keywords: Candida albicans, Candida dubliniensis, Detection, Differentiation, Duplex PCR

\footnotetext{
* Correspondence: suhail_ah@hsc.edu.kw

Department of Microbiology, Faculty of Medicine, Kuwait University, P. O.

Box 24923, Safat 13110, Kuwait
} 


\section{Background}

Candida albicans is the predominant pathogenic Candida species in susceptible human host. Infections caused by non-albicans Candida spp. have, however, also increased in recent years and may account for up to $60 \%$ of all episodes of candidemia or invasive candidiasis at some centers [1,2]. Candida dubliniensis, first described in 1995 from oral cavities of human immunodeficiency virus-infected individuals [3], is increasingly being reported from patients with candidemia in recent years [1,2,4]. C. dubliniensis shares several phenotypic characteristics, such as ability to form chlamydospores on cornmeal agar and germ tubes in serum with C. albicans. Since germ tube test is routinely used for the differentiation of $C$. albicans from other Candida species, identification based solely on this test leads to misidentification of some C. albicans isolates in routine diagnostic laboratories [5]. Accurate identification is warranted since $C$. dubliniensis exhibits increased adherence to buccal epithelial cells and is more likely to develop resistance against fluconazole and other azoles [6,7].

Several phenotypic tests based on colony morphology and physiological assimilation tests have been developed to distinguish $C$. albicans from $C$. dubliniensis. Commercially available yeast identification systems (Vitek 2 IDYST, API 20C and ID32C) based on utilization of various compounds have been used for differentiating various Candida spp., however, these methods are expensive and require at least 1-2 days to report results [8]. Formation of rough/fringed colonies by germ tube positive isolates on differential media such as Niger seed agar [9], simplified sunflower seed agar (SSA) [10], or tobacco agar [11], or inability to grow in a hypertonic Sabouraud dextrose broth containing $6.5 \mathrm{M} \mathrm{NaCl}$ [12] are often used to differentiate $C$. albicans from $C$. dubliniensis. A commercial latex agglutination test (Bichro-Dubli Fumouze) is also available [13]. However, prior presumptive identification of C. albicans/C. dubliniensis is required before phenotypic methods could be used for their differentiation. Also, variations in growth conditions (incubation temperature, repeated subculturing and storage) may impede accurate identification of these two species by phenotypic tests. Unambiguous identification can be established only by molecular techniques and PCR-based methods are mostly employed [5]. Matrix-assisted laser desorption ionizationtime of flight mass spectrometry (MALDI-TOF MS) has also been exploited recently for identification and differentiation of cultured yeast isolates including even closely related Candida spp. [14]. However, the high cost of the equipment is a major impediment for its widespread use in resource-limited settings. In this study, we have developed a novel duplex PCR assay by using primers derived from unique rDNA sequences for rapid detection and differentiation of $C$. albicans and C. dubliniensis. The performance of duplex PCR was compared with three phenotypic methods for identification of clinical isolates as C. albicans or C. dubliniensis.

\section{Methods}

Reference strains and clinical isolates

Candida dubliniensis (CD 36/CBS 7987/ATCC MYA646), C. albicans (ATCC 90028), Candida parapsilosis (ATCC 10233), Candida glabrata (ATCC 15545), Candida tropicalis (ATCC 750) and Candida krusei (CBS 6258) were used as reference Candida species. One hundred thirty-four clinical isolates representing C. dubliniensis $(\mathrm{n}=67), C$. albicans $(\mathrm{n}=55), C$. parapsilosis $(\mathrm{n}=2)$, C. glabrata $(\mathrm{n}=2)$, C. tropicalis $(\mathrm{n}=2)$, Candida lusitaniae $(\mathrm{n}=2)$, Candida krusei $(\mathrm{n}=2)$ and Candida kefyr $(\mathrm{n}=2)$ were tested by both phenotypic and molecular tests. All isolates of C. albicans and C. dubliniensis were positive by germ tube test. Identity of $C$. dubliniensis strains was also confirmed by sequencing of internal transcribed spacer (ITS) region (containing ITS-1, 5.8S rRNA and ITS-2) of rDNA as described previously [15]. Additionally, 239 clinical yeast isolates including C. albicans $(\mathrm{n}=148), C$. dubliniensis $(\mathrm{n}=50), C$. parapsilosis $(\mathrm{n}=5), C$. glabrata $(\mathrm{n}=5)$, C. lusitaniae $(\mathrm{n}=5)$, C. tropicalis $(\mathrm{n}=5)$, Candida haemulonii $(\mathrm{n}=3)$, Candida rugosa $(\mathrm{n}=2)$, Trichosporon species $(\mathrm{n}=4)$ and Cryptococcus species $(\mathrm{n}=6)$ and one isolate each of C. krusei, Candida guilliermondii, C. orthopsilosis, C. metapsilosis, C. nivariensis and C. bracarensis were also tested by duplex PCR. The isolates were cultured as part of standard patient care and no individual patient approval was needed for their subsequent use. The project under which the study was performed was approved by the Ethical Committee of Faculty of Medicine, Health Sciences center, Kuwait University.

\section{Phenotypic identification and characterization}

Clinical isolates were speciated by carbohydrate assimilation profile using Vitek 2 ID-YST system (bioMerieux, Marcy-lEtoile, France) and tested for germ tube formation in horse serum. All germ tube positive isolates were presumptively identified as C. albicans/C. dubliniensis. Germ tube positive isolates showing fringed/rough colonies on SSA [10] were identified as C. dubliniensis. BichroDubli (Fumouze Diagnotics, Levallois-Perret, France) latex agglutination test for identification of $C$. dubliniensis strains was performed on all germ tube positive isolates according to manufacturer's recommendations.

\section{Template DNA preparation, duplex PCR assay and DNA sequencing}

For molecular studies, a loop full of yeast colony from Sabouraud dextrose agar plate was suspended in $1 \mathrm{ml}$ of sterile water in a microcentrifuge tube containing $50 \mathrm{mg}$ 
Chelex-100 (Sigma-Aldrich Co.), the contents were heated at $95^{\circ} \mathrm{C}$ for $20 \mathrm{~min}$ and then centrifuged. The supernatant was transferred to a new tube and typically $2 \mu \mathrm{l}$ was used in PCR. Species-specific identity of C. albicans and C. dubliniensis strains was performed by duplex PCR by using primers targeting sequences in ITS-1 and ITS-2 regions of rDNA. Species-specificity of primer pairs CALF (5'-TGGTAAGGCGGGATCGCTT-3') + CALR (5'-GGTCAAAGTTTGAAGATATAC) and CDUF (5'-AAACTTGTCACGAGATTATTTTT) + CDUR (5'-AAAGTTTGAAGAATAAAATGGC-3') for C. albicans and $C$. dubliniensis, respectively, was indicated by BLAST searches (http://www.ncbi.nlm.nih.gov/ BLAST/Blast.cgi?). PCR amplification was performed in a final volume of $50 \mu \mathrm{l}$ containing $1 \mathrm{x}$ AmpliTaq DNA polymerase buffer I and 2 units of AmpliTaq DNA polymerase (Perkin Elmer), 10 pmol of CALF + CALR + CDUF + CDUR primers, $2 \mu \mathrm{l}$ of template DNA and $100 \mu \mathrm{M}$ of each dNTP. Cycling conditions included an initial denaturation at $95^{\circ} \mathrm{C}$ for $5 \mathrm{~min}$ followed by 30 cycles of $95^{\circ} \mathrm{C}$ for $1 \mathrm{~min}, 55^{\circ} \mathrm{C}$ for $30 \mathrm{~s}$ and $72^{\circ} \mathrm{C}$ for $1 \mathrm{~min}$ and a final extension at $72^{\circ} \mathrm{C}$ for $10 \mathrm{~min}$. PCR products $(20 \mu \mathrm{l})$ were run on $2 \%(\mathrm{w} / \mathrm{v})$ agarose gels, as described previously [16]. The species-specific identity of clinical yeast isolates was established by sequencing of ITS region of rDNA. The ITS region was amplified by using panfungal primers ITS1 [17] and CTSR [18] and the amplicons were purified and sequenced as described in detail previously $[15,19]$.

\section{Results}

Duplex PCR amplification with CALF + CALR + CDUF + CDUR primers yielded an amplicon of $\sim 100$ bp and $\sim 325$ bp only with DNA from reference strains of $C$. albicans and $C$. dubliniensis, respectively, but not from other Candida species, as expected (data not shown). Of 134 clinical Candida spp. isolates, 122 were positive for germ tube formation and 12 were germ tube negative. Vitek 2 ID-YST system identified the isolates as C. dubliniensis $(\mathrm{n}=67), C$. albicans $(\mathrm{n}=55), C$. parapsilosis $(\mathrm{n}=2)$, C. glabrata $(\mathrm{n}=2)$, C. tropicalis $(\mathrm{n}=2)$, C. lusitaniae $(\mathrm{n}=2)$, C. krusei $(\mathrm{n}=2)$ and C. kefyr $(\mathrm{n}=2)$. Duplex PCR correctly identified all 67 C. dubliniensis and 55 C. albicans isolates (data from 11 selected isolates are shown in Figure 1) while negative results were obtained for the remaining 12 germ tube negative isolates (Table 1). Growth on SSA correctly identified 67 of 122 germ tube positive isolates as $C$. dubliniensis. BichroDubli test was positive for 64 of 67 , weakly positive for 2 of 67 and negative for 1 of 67 isolates identified as $C$. dubliniensis by Vitek 2 ID-YST system and ITS region sequencing. Bichro-Dubli test was negative for 55 of 122 germ tube positive isolates identified as C. albicans by Vitek 2 ID-YST system as well as 12 germ tube negative

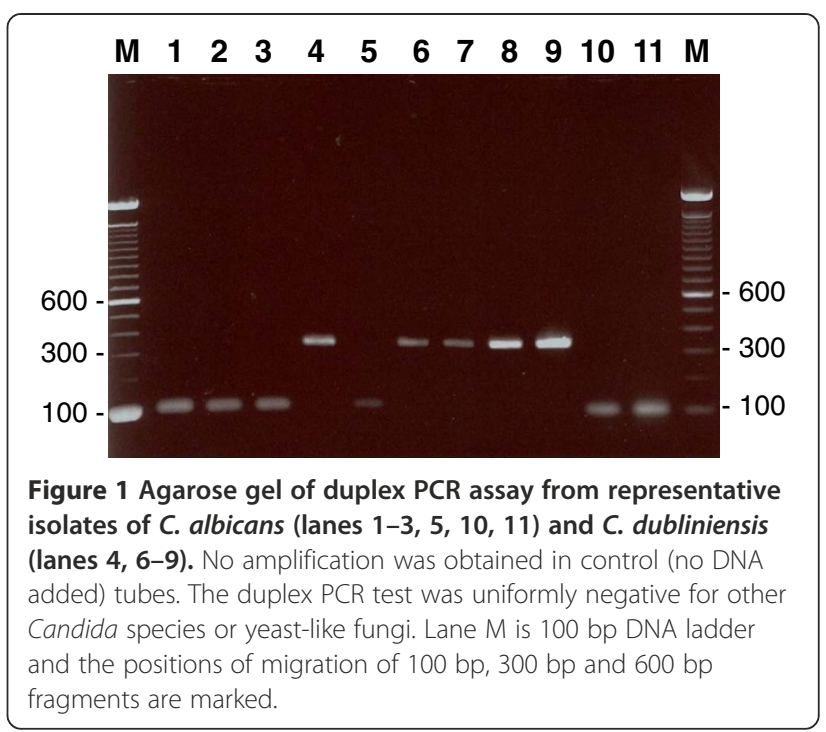

isolates of six other Candida species (Table 1). Thus, duplex PCR accurately identified all 55 C. albicans and 67 C. dubliniensis strains among a panel of 134 wellcharacterized Candida spp. isolates with 100\% sensitivity and specificity.

Direct application of duplex PCR on a panel of 239 clinical isolates of yeast-like fungi correctly identified all 148 C. albicans and 50 C. dublininsis strains. The remaining isolates representing other Candida spp. or other yeast-like fungi yielded negative results. The results were confirmed by direct DNA sequencing of ITS region of rDNA.

\section{Discussion}

The results of duplex PCR assay described here were completely concordant with species-specific identification of C. dubliniensis and C. albicans strains by Vitek 2 ID-YST system and colony characteristics (fringed/rough colonies) on SSA. However, both, Vitek 2 ID-YST system and growth on SSA usually require 24-48 hours before results are available. Furthermore, identification of $C$. dubliniensis strains on SSA requires prior testing for germ tube formation while Vitek 2 ID-YST system is expensive and thus, is not readily available in resourcepoor settings [10]. The requirement for minimal amount of genomic DNA for duplex PCR allows the whole procedure to be completed within 4 hours. Furthermore, since duplex PCR employs species-specific primers for both, $C$. albicans and $C$. dubliniensis, it can be directly applied on clinical isolates of yeast and yeast-like fungi. Indeed, direct application of duplex PCR on a panel of 239 clinical isolates of yeast-like fungi correctly identified all 50 C. dublininsis and 148 C. albicans strains. 
Table 1 Performance comparison of duplex PCR with Vitek 2 ID-YST system, Bichro-Dubli Fumouze latex agglutination test and colony characteristics on simplified sunflower seed agar (SSA) for identification and differentiation of $C$. albicans and C. dubliniensis

\begin{tabular}{|c|c|c|c|c|c|}
\hline \multirow[t]{2}{*}{ Candida species } & \multirow{2}{*}{$\begin{array}{l}\text { No. of isolates } \\
\text { tested }\end{array}$} & \multicolumn{4}{|c|}{ No. of isolates yielding correct identification with } \\
\hline & & Vitek 2 ID-YST & SSA* $^{*}$ & Bichro-Dubli & Duplex PCR \\
\hline C. albicans & 55 & 55 & 0 & 0 & 55 \\
\hline C. dubliniensis & 67 & 67 & 67 & 66 & 67 \\
\hline C. glabrata & 2 & 2 & Not done & 0 & 0 \\
\hline C. parapsilosis & 2 & 2 & Not done & 0 & 0 \\
\hline C. tropicalis & 2 & 2 & Not done & 0 & 0 \\
\hline C. lusitaniae & 2 & 2 & Not done & 0 & 0 \\
\hline C. krusei & 2 & 2 & Not done & 0 & 0 \\
\hline C. kefyr & 2 & 2 & Not done & 0 & 0 \\
\hline
\end{tabular}

*Fringed/rough colonies during growth on simplified sunflower seed agar (SSA).

Although several PCR-based methods using rDNA as target have been described previously, they either involve two separate PCR reactions for each strain $[16,20,21]$ or further manipulations (such as restriction digestion to generate restriction fragment length polymorphism or DNA sequencing) are needed for species-specific identification [16,22-24]. These additional steps add to the cost of the test and/or consume additional time thus delaying results. Other investigators have used PCRbased methods targeting intronic sequences for differentiation of C. albicans from C. dubliniensis [25-27]. However, these approaches may lead to misidentification of some isolates since intronic sequences may vary significantly [27]. A real-time PCR assay using melting point analysis was described recently for detection and differentiation of $C$. albicans and $C$. dubliniensis strains but involves separate reactions for each Candida spp. and expensive probe primers [28]. Although two realtime PCR assays using SYBR Green dye have also been described, these assays either require prior presumptive identification of $C$. albicans/C. dubliniensis strains by another (such as germ tube) test and/or two rounds of real-time PCR assays are needed [29,30]. Furthermore, the difference in $T_{m}$ values for $C$. albicans and $C$. dubliniensis strains in the two reports was either small $\left(<0.5^{\circ} \mathrm{C}\right)$ or negligible which may lead to misidentification of some isolates.

Performance of another rapid (Bichro-Dubli Fumouze latex agglutination) test was also compared with duplex PCR results. The $98.5 \%$ sensitivity and specificity of $100 \%$ of Bichro-Dubli test is in agreement with the reported sensitivity (97\% to $100 \%$ ) and specificity (100\%) values suggested by the kit manufacturer. Similar values have also been reported in other studies for identification of C. dubliniensis isolates [31,32]. Although BichroDubli test is also a rapid method for identification of C. dubliniensis, the test can only be applied on isolates previously identified as C. albicans/C. dubliniensis either by germ tube formation of by application of another (Bichro Latex Albicans) latex agglutination test [13,32]. This increases both, the cost and the time to report results for Bichro-Dubli test. The use of chromogenic differential media such as CHROMagar ${ }^{\circledR}$ Candida has also been implemented in many clinical microbiology laboratories for identification and differentiation of both C. albicans and $C$. dubliniensis, thus diminishing the need for germ tube test. However, the production of light green (for C. albicans) or dark green (for C. dubliniensis) color is inconsistent when large number of isolates are screened, thus requiring further cultivation on a bird-seed agar medium for their accurate identification [33].

\section{Conclusions}

A duplex PCR assay has been developed and extensively evaluated for the detection and differentiation of clinical isolates as C. dubliniensis or C. albicans. The method may be applied directly on clinical yeast isolates for their identification as C. dubliniensis or C. albicans as it does not require prior testing for germ tube formation or other such tests and is suitable for resource-limited clinical microbiology laboratories equipped with basic PCR technology. A limitation of the study is that other Candida and yeast-like organisms will require additional testing for their species-specific identification.

\section{Abbreviations \\ ITS: Internal transcribed spacer; SSA: Simplified sunflower seed agar; rDNA: Ribosomal DNA.}

\section{Competing interests}

The authors declare that no competing interests exist.

\section{Authors' contributions}

SA and ZK designed the study, arranged financial support and analyzed the data. MA, AT and RC performed the experiments. All authors contributed in writing the manuscript and approved the final version. 


\section{Acknowledgments}

The study was supported by KURA grant no. MI01/08

Received: 10 June 2012 Accepted: 24 September 2012 Published: 25 September 2012

\section{References}

1. Horn DL, Neofytos D, Anaissie EJ, Fishman JA, Steinbach WJ, Olyaei AJ, Marr KA, Pfaller MA, Chang CH, Webster KM: Epidemiology and outcomes of candidemia in 2019 patients: data from the prospective antifungal therapy alliance registry. Clin Infect Dis 2009, 48:1695-1703.

2. Miceli MH, Díaz JA, Lee SA: Emerging opportunistic yeast infections. Lancet Infect Dis 2011, 11:142-151.

3. Sullivan DJ, Westerneng TJ, Haynes KA, Bennett DE, Coleman DC: Candida dubliniensis sp. nov.: phenotypic and molecular characterization of a novel species associated with oral candidosis in HIV-infected individuals. Microbiology 1995, 141:1507-1521.

4. Khan Z, Ahmad S, Joseph L, Chandy R: Candida dubliniensis: an appraisal of its clinical significance as a bloodstream pathogen. PLoS One 2012, 7:e32952.

5. Ells R, Kock JLF, Pohl CH: Candida albicans or Candida dubliniensis. Mycoses 2011, 54:1-16.

6. Pinjon E, Moran GP, Coleman DC, Sullivan DJ: Azole susceptibility and resistance in Candida dubliniensis. Biochem Soc Trans 2003, 33:1210-1214.

7. Fleischhacker M, Prasligh J, Moran G, Ruhnke M: Longitudinal genotyping of Candida dubliniensis isolates reveals strain maintenance, microevolution, and the emergence of itraconazole resistance. J Clin Microbiol 2010, 48:1643-1650.

8. Pincus DH, Coleman DC, Pruitt WR, Padhye AA, Salkin IF, Geimer M, Bassel A, Sullivan DJ, Clarke M, Hearn V: Rapid identification of Candida dubliniensis with commercial yeast identification systems. J Clin Microbiol 1999, 37:3533-3539.

9. Al-Mosaid A, Sullivan D, Salkin IF, Shanley D, Coleman DC: Differentiation of Candida dubliniensis from Candida albicans on staib agar and caffeic acid-ferric citrate agar. J Clin Microbiol 2001, 39:323-327.

10. Khan ZU, Ahmad S, Mokaddas E, Chandy R: Simplified sunflower (Helianthus annuus) seed agar for differentiation of Candida dubliniensis from Candida albicans. Clin Microbiol Infect 2004, 10:590-592.

11. Khan ZU, Ahmad S, Mokaddas E, Chandy R: Tobacco agar, a new medium for differentiating Candida dubliniensis from Candida albicans. J Clin Microbiol 2004, 42:4796-4798.

12. Alves SH, Milan EP, de Laet Sant'Ana P, Oliveira LO, Santurio JM, Colombo $\mathrm{AL}$ : Hypertonic sabouraud broth as a simple and powerful test for Candida dubliniensis screening. Diagn Microbiol Infect Dis 2002, 43:85-86.

13. Marot-Leblond A, Beucher B, David S, Nail-Billaud S, Robert R: Development and evaluation of a rapid latex agglutination test using monoclonal antibody to identify Candida dubliniensis colonies. J Clin Microbiol 2006, 44:138-142.

14. Dhiman N, Hall L, Wohlfiel SL, Buckwalter SP, Wengenack NL: Performance and cost analysis of matrix-assisted laser desorption ionization-time of flight mass spectrometry for routine identification of yeast. J Clin Microbiol 2011, 49:1614-1616.

15. Al-Sweih N, Ahmad S, Khan ZU, Khan S, Chandy R: Prevalence of Candida dubliniensis among germ tube-positive Candida isolates in a maternity hospital in Kuwait. Mycoses 2005, 48:347-351.

16. Ahmad S, Khan Z, Mokaddas E, Khan ZU: Isolation and molecular identification of Candida dubliniensis from non-human immunodeficiency virus-infected patients in Kuwait. J Med Microbio/ 2004, 56:633-637.

17. White TJ, Burns TD, Lee SB, Taylor JW: Amplification and direct sequencing of fungal ribosomal RNA genes for phylogenetics. In PCR protocols: a guide to methods and applications. Edited by Innis MA, Gelfand $\mathrm{GH}$, Sninsky JJ, White TJ. San Diego: Academic; 1990:315-322.

18. Ahmad S, Khan Z, Mustafa AS, Khan ZU: Semi-nested PCR for diagnosis of candidemia: comparison with culture, antigen detection, and biochemical methods for species identification. J Clin Microbiol 2002, 40:2483-2489

19. Ahmad S, Khan ZU, Joseph L, Asadzadeh M, Theyyathel A: Genotypic heterogeneity and molecular basis of 5-flucytosine resistance among Candida dubliniensis isolates recovered from clinical specimens in Kuwait. Med Mycol 2012, 50:244-251.
20. Lau A, Halliday C, Chen SC, Playford EG, Stanley K, Sorrell TC: Comparison of whole blood, serum and plasma for early detection of candidemia by multiplex-tandem PCR. J Clin Microbiol 2010, 48:811-816.

21. Lung Than TL, Chong PP, Ng KP, Seow HF: Detection of 10 medically important Candida species by seminested polymerase chain reaction. Diagn Microbiol Infect Dis 2011, 72:196-198.

22. Williams DW, Coulter WA, Wilson MJ, Potts AJ, Lewis MA: Identification of Candida dubliniensis, based on ribosomal DNA sequence analysis. $\mathrm{Br} J$ Biomed Sci 2001, 58:11-16.

23. Graf B, Trost A, Eucker J, Gobel UB, Adam T: Rapid and simple differentiation of Candida dubliniensis from Candida albicans. Diagn Microbiol Infect Dis 2004, 48:149-151.

24. Romeo O, Racco C, Criseo G: Amplification of the hyphal wall protein 1 gene to distinguish Candida albicans from Candida dubliniensis. J Clin Microbiol 2006, 44:2590-2592.

25. Romeo O, Criseo G: First molecular method for discriminating between Candida africana, Candida albicans and Candida dubliniensis by using hwp1 gene. Diagn Microbiol Infect Dis 2008, 62:230-233.

26. Bosco-Borgeat ME, Taverna CG, Cordoba S, Isla MG, Murisengo OA, Szusz W, Vivot W, Davel G: Prevalence of Candida dubliniensis fungemia in Argentina: identification by a novel multiplex PCR and comparison of different phenotypic methods. Mycopathologia 2011, 172:407-414.

27. Riberio PM, Querido SM, Back-Brito GN, Mota AJ, Koga-Ito CY, Jorge AO: Research on Candida dubliniensis in a Brazilian yeast collection obtained from cardiac transplant, tuberculosis, and HIV-positive patients, and evaluation of phenotypic tests using agar screening methods. Diagn Microbiol Infect Dis 2011, 71:81-86.

28. Fricke S, Fricke C, Schimmelpfennig C, Oelkrug C, Schonfelder U, Blatz R, Zilch C, Faber S, Hilger N, Ruhnke M, Rodloff AC: A real-time PCR assay for the differentiation of Candida species. J Appl Microbiol 2010, 109:1150-1158

29. Somogyvari F, Doczi I, Serly J, Ahmad S, Nagy E: Rapid discrimination between Candida albicans and Candida dubliniensis by using real-time polymerase chain reaction. Diagn Microbiol Infect Dis 2007, 58:367-369.

30. Khan ZU, Mustafa AS, Alam FF: Real-time LightCycler polymerase chain reaction and melting temperature analysis for differentiation of clinically important Candida species. J Microbiol Immunol Infect 2009, 42:290-295.

31. Sahand IH, Moragues MD, Robert R, Quindos G, Ponton J: Evaluation of Bichro-Dubli Fumouze ${ }^{\circledR}$ to distinguish Candida dubliniensis from Candida albicans. Diagn Microbiol Infect Dis 2006, 55:165-167.

32. Chryssanthou E, Fernandez V, Petrini B: Performance of commercial latex agglutination tests for the differentiation of Candida dubliniensis and Candida albicans in routine diagnostics. APMIS 2007, 115:1281-1284.

33. Pasligh J, Radecke C, Fleischhacker M, Ruhnke M: Comparison of phenotypic methods for the identification of Candida dubliniensis. J Microbiol Immunol Infect 2010, 43:147-154.

doi:10.1186/1471-2334-12-230

Cite this article as: Ahmad et al.: Performance comparison of phenotypic and molecular methods for detection and differentiation of Candida albicans and Candida dubliniensis. BMC Infectious Diseases 2012 $12: 230$.

\section{Submit your next manuscript to BioMed Central and take full advantage of:}

- Convenient online submission

- Thorough peer review

- No space constraints or color figure charges

- Immediate publication on acceptance

- Inclusion in PubMed, CAS, Scopus and Google Scholar

- Research which is freely available for redistribution 